\title{
COMBINED PULLEY-COUNTERWEIGHT SYSTEM FOR UPLIFT AND INCLINATION MECHANISM OF A CHAIR'S SEAT
}

\author{
Tara Agarwal \\ PDMDS - Parkinson's Disease and Movement Disorders Society, \\ Mumbai, India \\ tara.agarwal2@gmail.com
}

\begin{abstract}
This paper addresses the challenges experienced by people with motor disabilities and movement disorders like Parkinson's Disease while independently standing from a seated position on a chair. The paper presents a solution to allow for the inclination of a chair's seat, therefore facilitating the user's sit-to-stand and stand-to-sit motion. The design put forward involves the use of a combined pulley mechanism to lift the seat and consequently support the user, apart from employing a counterweight to restore equilibrium. The paper explores the construction, optimum materials and dimensions of each mechanical component through the use of foundational principles of mathematics and physics. It also outlines the operation of the mechanism as a whole, aided by visuals including three-dimensional computer aided design renders and free body diagrams. Having taken into consideration the disability-poverty nexus, the mechanism proposed deviates from the use of electronic components, making it relatively inexpensive, easy to construct and thus accessible. The paper concludes by highlighting scope for future improvement of the system.
\end{abstract}

Keywords: chair egress, pulley-counterweight, uplift mechanism.

Cite this Article: Tara Agarwal, Combined Pulley-Counterweight System for Uplift and Inclination Mechanism of a Chair's Seat. International Journal of Mechanical Engineering and Technology. 11(7), 2020, pp. 1-6.

https://iaeme.com/Home/issue/IJMET?Volume $=11 \&$ Issue $=7$

\section{INTRODUCTION}

According to the World Bank and World Health Organisation Joint Report on Disability, approximately 200 million people across the globe, or $1 / 5^{\text {th }}$ of the total disabled population, live with significant disabilities including motor disorders and impairments like Parkinson's disease, muscular dystrophy and multiple sclerosis - all conditions that are characterized by limited muscle functioning and control.[1] Motor impairment, an aspect of physical disability, involves muscular weakness and fatigue, limiting individuals' range of motion and consequently their activity. As populations continue to age, the number of individuals suffering from the aforementioned disorders is expected to grow rapidly. 
Symptoms of many such muscular impairments manifest in the lower body and limbs, resulting in individuals with motor disabilities finding it difficult or nearly impossible to stand up from a seated position on a chair. This results in them relying on helpers or family members for movement, depriving them of a sense of independence and self-sufficiency and hampering their activity and lifestyle. Therefore, patients' inability to stand up from a sitting position leaves them with no alternative but to decidedly limit their movement or to continuously ask for assistance from passers-by: a difficulty clouded by stigma and a threat to safety as passers-by are likely to be unaware of the minutiae of the person's condition. Additionally, the deeply intertwined poverty-disability nexus suggests that many disabled individuals are unable to afford hiring helpers to assist their movement.

\section{THE MECHANISM}

Considering the difficulty faced by disabled persons in independently rising from conventional chairs, an uplift and inclination mechanism of a chair's seat that enables individuals to stand up and sit down without the need for external help is required. The system should be self-sufficient and be constructed primarily using low-cost mechanical components. It is also crucial that the designed mechanism takes into account safety considerations to ensure that sudden uplifts do not pose health hazards to the user. This paper illustrates the construction and testing of such a mechanism.

While some attempt has been made in the past to develop an attachable and portable egress cushion, a significant shortcoming of this is the limited range of motion it offers, drawing attention to the need for an uplift and inclination mechanism that provides greater support for the user.

\section{DESIGN AND CONSTRUCTION}

\subsection{Components}

Pulleys have been used for many centuries to lift heavy weights. Drawing inspiration from this application, the proposed design consists of a counterweight and pulley mechanism used to uplift the chair's seat when the user attempts to stand, yielding an inclined seat when the user is not seated, therefore also enabling them to apply less pressure on the knee joints while attempting to sit. The design involves the following mechanical components:

- Pulley System: A one string-7 pulley system (the reason for this is outlined in section 3.2) will be used to initiate incline of the seat as the person attempts to stand and decline as they sit. This will allow for the effect of the motion of the counterweight to influence the inclination of the seat.

- Counterweight: The counterweight balances the system and is attached via a rope or wire to the pulley below the seat. It moves downwards as the person attempts to stand, causing the seat to steadily incline upwards and therefore facilitating the sit-to-stand motion. The counterweight restores the system's equilibrium in light of the person's movement.

- Steel rods: Two steel rods are required to connect the subsidiary pulleys to those attached to the frame of the chair. One rod will be fixed to the top of the chair's frame and another to the rear end of the seat.

- Hinges: The seat should be connected to the front legs using hinges, which will allow for the incline and decline of the seat. The joint shall allow for a range movement of 0 to 45 degrees above the horizontal axis parallel to the seat. 


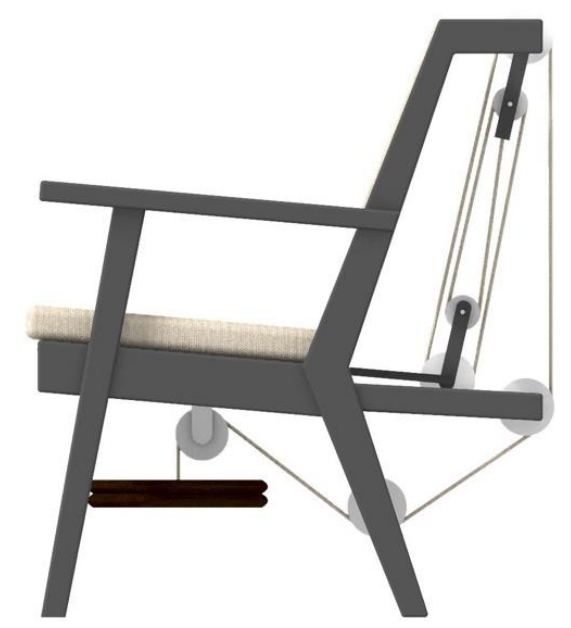

Figure 1 2D Render of the Proposed Mechanism

\subsection{Determination of Counterweight's Mass}

For the following calculations, the gravitational field strength is considered to be $9.81 \mathrm{~ms}^{-2}$. The weight of a seated person on the chair's cushion(seat) is determined to be $78 \%$ of total body weight based on a study by the Association for Advancement of Automotive Medicine.[2]

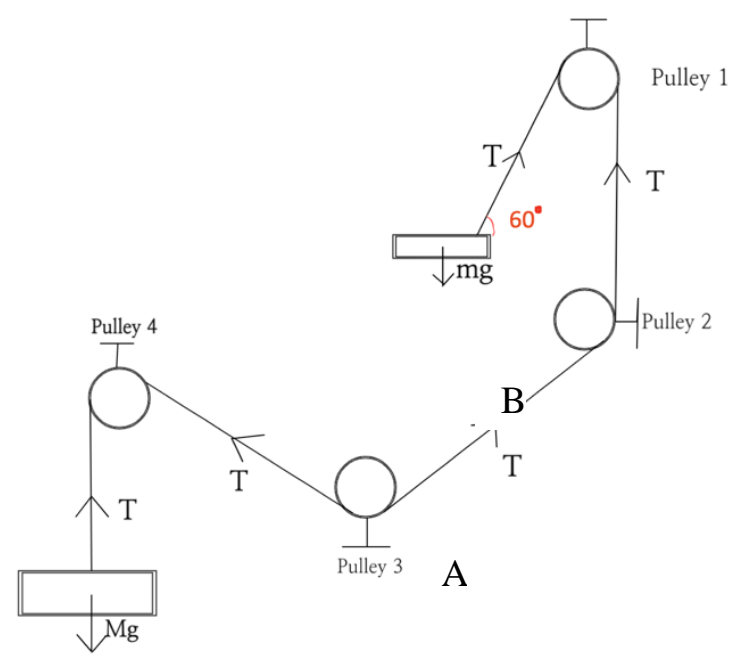

Figure 2 Free Body Diagram 4 Pulley System

Initially the design considered involved the use of four pulleys, as depicted in Figure 2. Based on this the counterweight's mass was calculated as follows:

Using Fig 1:

$$
\begin{aligned}
T \sin 60 & =0.78 \cdot 70 \cdot 9.81 \\
\mathrm{~T} & =618.5 \mathrm{~N}
\end{aligned}
$$

The mass, $\mathrm{M}$, of the counterweight can thus be calculated as:

$$
\begin{gathered}
M g=T \\
\Rightarrow M=63.04 \mathrm{~kg}
\end{gathered}
$$

However, this value of $\mathrm{M}$ would result in the chair becoming very heavy, difficult to construct and would also increase the likelihood of it tipping over as the center of mass moves forwards. By adding an additional pulley in distance $\mathrm{AB}$ (Figure 2), the required mass of the counterweight can be reduced, therefore yielding a new setup depicted in Figure 3. 
Using this system $M^{\prime}$, the revised necessary mass

of the counterweight is:

$$
\begin{aligned}
M^{\prime} & =\frac{T}{2 g} \\
M^{\prime} & =31.5 \mathrm{~kg}
\end{aligned}
$$

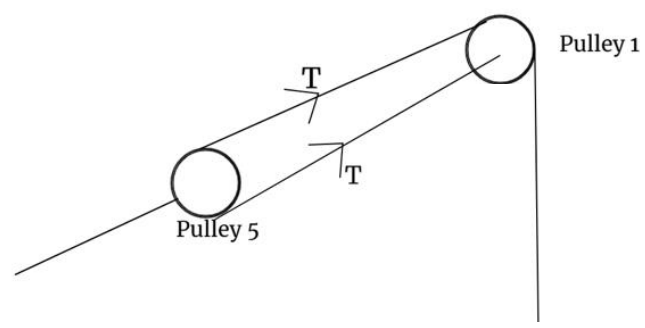

Figure 3 Line Diagram 5 Pulley System

The system can be further modified to reduce the necessary mass of the counterweight by adding 2 pulleys between A and B of Fig 3 as depicted in Figure 1. Using this system $M^{\prime \prime}$, the revised necessary mass of the counterweight is:

$$
\begin{gathered}
M^{\prime \prime}=\frac{T}{4 g} \\
M^{\prime \prime}=15.8 \mathrm{~kg}
\end{gathered}
$$

While the mechanism can be continually revised to reduce the required mass of the counterweight by adding more pulleys, this can lead to cluttering of the mechanism. Therefore, this paper and the associated design considers a 7-pulley system as depicted in Figure 1.

\subsection{Materials and Dimensions}

\subsubsection{Pulley Mechanism}

The rope or wire used in the pulley system had to have a tensile strength that was great enough to withstand the tension calculated in section 3.2 of approximately $618.5 \mathrm{~N}$. It should also be thin enough to be strung through the pulley. Three materials nylon[3], wire rope[4] and terylene[5] were considered for the rope of the pulley. Once the material was selected, the diameter was chosen based on the lowest alternative to allow for an appropriate safety limit.

Table 1

\begin{tabular}{|l|c|c|c|}
\hline \multicolumn{1}{|c|}{ Material } & Diameter(mm) & Mass(kg/m) & Safe Load(kN) \\
\hline Nylon & 10 & 0.053 & 1.20 \\
\hline Wire Rope & 6.4 & 0.16 & 4.89 \\
\hline Terylene (dacron) & 10 & 0.065 & 1.07 \\
\hline
\end{tabular}

The masses of the ropes are almost negligible in comparison to the mass of the person and the counterweight. Thus, the determination of the ideal material was not based on the mass of the rope. The elasticity of nylon can result in the load, in this case the user, shaking which is a safety hazard. Therefore, the use of nylon was eliminated. Between terylene and wire rope, wire rope offers considerable advantages by supporting a much larger safe load with a significantly smaller diameter. Thus, it was decided to use wire rope made of steel. (While the 
term 'wire rope' applies specifically to a diameter larger than $9.52 \mathrm{~cm}$, the nature of its usage in this context deems the term appropriate.)

\subsubsection{Construction Dimensions}

The dimensions for the base chair are a standard depth of $46 \mathrm{~cm}$, a distance of $52 \mathrm{~cm}$ between the rare and front legs and a total height of $96 \mathrm{~cm}$. This yields a backrest length of $51 \mathrm{~cm}$. The length of wire required can thus be calculated using Fig 1, 3 and scale diagram to be $268 \pm 2$ $\mathrm{cm}$.

\section{OPERATION}

\section{Sit-to-stand motion:}

- The user is seated on the chair. The rope of the pulley is taut and the counterweight has risen upwards and is unmoving, as depicted in Figure 5 below.

- The user attempts to stand by shifting their body weight and consequently the center of mass slightly forwards.

- The force exerted by the user on the seat decreases, causing the tension in the pulley's rope to decrease.

- The counterweight moves downwards towards the floor restoring equilibrium, and the seat of the chair is inclined so as to support the user in their sit-to-stand motion by providing the necessary upward thrust. The seat is unable to move beyond a 45 degree angle due to the limited rotation provided by the hinge at the fore legs. This has been depicted in Figure 6 below.

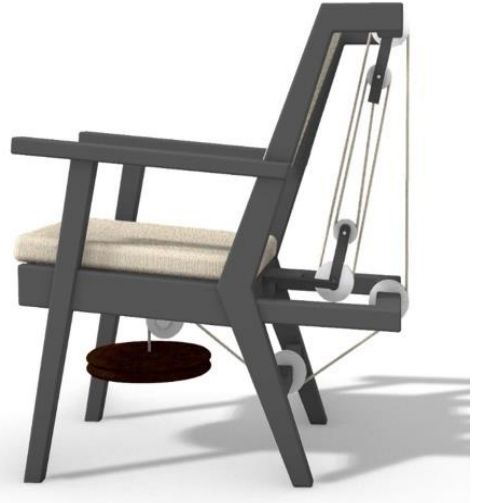

Figure 4 3D render of the chair when person is seated

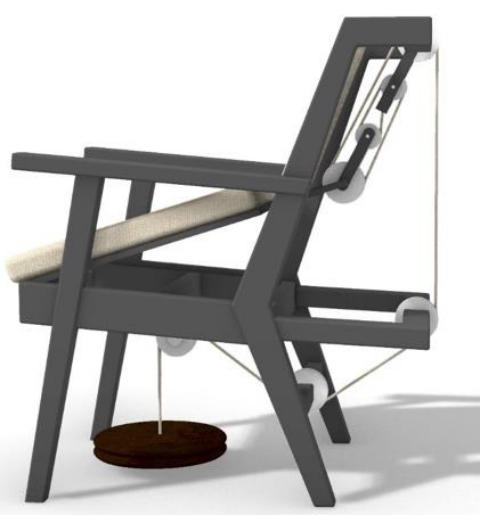

Figure 5 3D render of the inclined seat mechanism

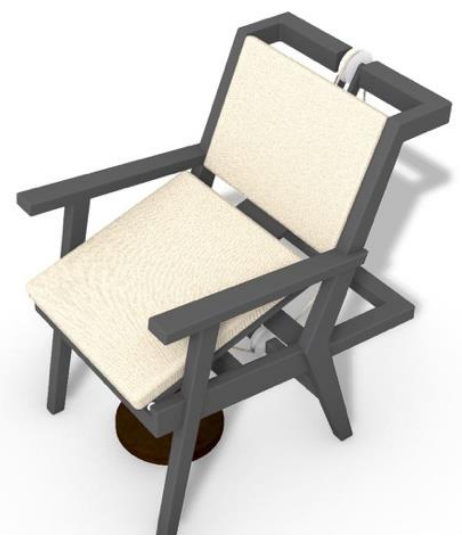

Figure 6 3D Render of the chair from a height

\section{Stand-to-sit motion:}

- The chair's seat is already inclined.

- The user bends downwards to apply some weight on the chair, causing the tension in the string to increase.

- The counterweight moves upwards and the seat moves downwards as the person bends lower to facilitate the stand-to-sit motion. 


\section{EVALUATION AND SCOPE}

- A limitation of this design is that it cannot be adapted to fit all chairs as it requires the seat to be separate from the base-rest. Therefore, further scope for this design involves enhancing it to serve as a modification to pre-existing chairs rather than requiring the creation of a new one from scratch.

- There are also some concerns associated with the use of a wire rope which is likely to experience abrasion due to its movement through the pulley systems.

- The proposed chair is considerably heavier than conventional chairs due to the use of a $15.8 \mathrm{~kg}$ counterweight. Future developments of this design could possibly work towards reducing the net weight of the chair through design modifications such as pulley additions.

\section{CONCLUSION}

The design discussed in the paper is relatively inexpensive, catering to the target user, while also being effective in inclining the seat. The mechanism does not require any additional force from the user and once triggered occurs independently. The design takes into account the safety of the user by providing extensive side handles that can be held onto for additional support apart from providing a pre-set maximum angle of incline considered to be safe. The paper discusses the individual components of the set-up and their purposes, the construction, weight and material considerations apart from providing 3D renders to aid the understanding of its operation.

\section{REFERENCES}

[1] World Report on Disabilities. WHO, 2011, https://www.who.int/disabilities/world_report/2011/report.pdf?ua=1

[2] W. Shen, C. Parenteau, R. Roychoudhury and J. Robbins, Seated Weight Distribution of Adults and Children in Normal and Non-Normal Positions, Association for Advancement of Automotive Medicine

[3] "Nylon Rope Strength" Engineering Toolbox.com https://www.engineeringtoolbox.com/nylon-rope-strength-d_1513.html

[4] "Wire Rope Stength" Engineering Toolbox.com https://www.engineeringtoolbox.com/wire-rope-strength-d_1518.html

[5] "Polyester Rope Strength" Engineering Toolbox.com https://www.engineeringtoolbox.com/polyester-rope-strength-d_1514.html 